\title{
How Accurate Must Potentials Be for Successful Modeling of Protein Folding?
}

\author{
Vijay S. Pande, Alexander Yu. Grosberg†, Toyoichi Tanaka \\ Department of Physics and Center for Materials Science and Engineering, \\ Massachusetts Institute of Technology, Cambridge, Massachusetts 02139, USA \\ †On leave from: Institute of Chemical Physics, \\ Russian Academy of Sciences, Moscow 117977, Russia
}

(September 25, 2018)

\begin{abstract}
Protein sequences are believed to have been selected to provide the stability of, and reliable renaturation to, an encoded unique spatial fold. In recently proposed theoretical schemes, this selection is modeled as "minimal frustration," or "optimal energy" of the desirable target conformation over all possible sequences, such that the "design" of the sequence is governed by the interactions between monomers. With replica mean field theory, we examine the possibility to reconstruct the renaturation, or freezing transition, of the "designed" heteropolymer given the inevitable errors in the determination of interaction energies, that is, the difference between sets (matrices) of interactions governing chain design and conformations, respectively. We find that the possibility of folding to the designed conformation is controlled by the correlations of the elements of the design and renaturation interaction matrices; unlike random heteropolymers, the ground state of designed heteropolymers is sufficiently stable, such that even a substantial error in the interaction energy should still yield correct renaturation.
\end{abstract}

To appear in Journal of Chemical Physics, December 1995 


\section{INTRODUCTION}

\section{A. What is this work about?}

The native state of a protein is in a sense "written" in the sequence using the "language" of physical interactions between monomers. In this work, we examine the effects of "misunderstandings" and "misspellings" of this language.

A somewhat related question was recently discussed by Bryngelson [1]. He considered heteropolymer chains with random sequence and estimated the probability that its lowest energy conformation will be correctly detailed by the model with noisy distorted potentials of volume interactions between monomers. The result is that the probability, $p$, diminishes with noise amplitude, $\eta$, as $p \sim 1-$ const $\cdot \eta N^{1 / 2}$; for sufficiently long chain, or in thermodynamic limit, there is no chance to compute equilibrium conformation given that some mistakes in the determination of energies are inevitable.

By contrast, we consider here heteropolymer chains with sequences that are not random, but rather "designed" [2], or "imprinted" [3], or "selected" [4], or, in other words, obey the so-called principle of minimal frustration [5]. We show, that for these chains the situation is dramatically different, and there is finite probability of successful recovery of thermodynamically stable conformation, even in thermodynamic limit $(N \rightarrow \infty)$ and for finite non-vanishing $\eta$.

As the work is based on rather heavy theoretical machinery, we begin with more general introduction.

\section{B. Protein folding in a statistical mechanics perspective: a brief summary}

Protein folding is one of the great challenges of modern biophysics. Recently, there has been much progress and insight into protein folding from the statistical physics perspective. This came mainly with the ideas borrowed from the physics of disordered systems, such as spin glasses [6]. It is now widely believed that folding of a protein chain can be viewed as a 
freezing phase transition, as in spin glasses. The sequence of types of monomers along the chain leads to quenched disorder, and polymer bonds between monomers impose frustrations. The concept of a freezing transition seems to resolve one of the mysteries surrounding the problem, namely, why the native state of a protein is realized via a unique conformation, at least, in a coarse grained sense.

One of the main differences between proteins (and biopolymers in general) and other systems more familiar to physicists is the way in which quenched disorder appears. While in regular spin glasses and similar "un-animated" systems, the appearance of any particular realization of the disorder is far from any control, in biology the situation is dramatically different. First, the biosynthesis of proteins (as well as other biopolymers) manages to produce macroscopic amount of identical copies — a situation unthinkable in other disordered systems. Furthermore, the sequences of the existing real proteins are believed to be the product of evolution and thus are of great interest. Do they have some distinct properties compared to other possible sequences? Do they provide some general properties of proteins, such as their ability to form the unique spatial fold, or only particular conformation and/or function of each particular protein?

To this end, it now seems well established that the ability of polymer chain to undergo a freezing phase transition into the state with a unique (or almost unique) conformation is common to many models of random heteropolymers. In other words, to achieve the uniqueness of the ground state conformation one does not have to impose any requirements on the sequence, which can be therefore chosen even at random. This was first shown for the so-called independent-interaction model [7,8] (which is in a sense even more random than random heteropolymer: instead of $N$ independent monomers, it has about $\sim N^{2}$ independent monomer-to-monomer interaction constants). This argument was extended for random copolymers with two types of monomers [9] and brought into the most general form in Ref. [10].

In independent (and even earlier) development, the hypothesis was made that the sequences of real proteins deviate from random in such a way that to satisfy the requirement 
of the so-called "minimal frustration principle" [5]. In a sense, this idea goes back to even earlier ideas of Abe [11]. Recent developments, related mainly to computer Monte Carlo simulations of freezing kinetics, reveal the insight into the role of minimal frustration as a factor pulling down the energy of the ground state conformation and thus providing the gap in the energy spectrum necessary for reliable folding [2].

Thus, it seems that kinetic reliability of folding requires that sequences of proteins are not random, but "edited." Statistical analysis reveals indeed, that although the sequences are close to random [12], the systematic deviations from randomness do exist [13], and they are at least compatible with the idea of energy optimization of the ground state.

The question appears how to model the ensemble of sequences more realistically then by taking them at random. Two approaches has been recently suggested. Both employ physical interactions between monomers to build up polymeric sequence, and are both based on energy optimization of the native conformation. The first approach, due to Shakhnovich and Gutin [2], implies the search for optimization in "sequence" space by swapping the monomers along the chain while preserving (native) conformation. Apart from the speculative possibility to model evolution, this is obviously intended for computer simulation. On the other hand, we suggested energy optimization in the "monomer soup" prior to polymerization [3]. Although these two models are considerably different in spirit, they appear to be identical from the point of view of mean-field theoretical treatment.

The freezing transition of an ensemble of sequences which have been energetically optimized ("designed") for a particular conformation was examined for the black-and-white model (with two types of monomers) in Refs. [14.15]; the analysis was extended for the general case in Ref. [16]. For what follows, it is important that three globular (that is, compact) phases were found on the phase diagram of thus "designed" or "imprinted" polymer: we call them random, frozen and target. The random globular phase is pretty much the same as the globule of a homopolymer, it is comprised of vast number of conformations (all of which are compact). By contrast, frozen and target phases are each comprised of one or very few conformations. In the target phase, this unique conformation is exactly the one which is 
targeted by the design procedure. On the other hand, in the frozen phase, the system freezes to the conformation which is unrelated to the design conformation and therefore cannot be controlled.

\section{Sequence design and folding are governed by different interactions}

As sequence design is based on energy optimization, it employs physical interactions between monomers. It is however possible, and, moreover, almost inevitable, that these interactions are somewhat different from those governing folding. Apart from speculations on the interactions that governed the "design" of modern proteins by evolution, we mention three illustrations of our thesis:

1. When one tries to find theoretically or computationally the native state for a chain with a given sequence (direct protein folding problem), one can say that nature details the interactions used in the design of protein sequences and man-made potentials are used as substitutes in the simulations of renaturation.

2. Similarly, when one is looking for a sequence to fold into a given conformation, one is essentially trying to design the sequence using artificial potentials in such a way, that this sequence under real natural interactions will fold in a desirable way.

3. Speaking of the attempt to reproduce protein-like properties in the man made heteropolymer via the Imprinting procedure [3], we have to acknowledge some difference between interactions of monomers in the soup prior to polymerization and interactions of the links of polymer.

4. One can consider the renaturation of a protein in a solvent different than that used during "design" also as an experiment in which the interactions during design and renaturation are different.

If there are, say, $q$, different monomeric species involved in our polymer ( $q=20$ for proteins), interactions between species $i$ and $j$ can be described in terms of the $q \times q$ matrix $B_{i j}$. In 
general, there are two different matrices, $B_{i j}^{p}$ and $B_{i j}$ : the first governing preparation and second governing folding behavior of the already prepared chain.

To have two different interaction matrices for design and renaturation is somewhat similar to writer and reader who use different languages. Naprimer, my nadeemsya, chto nash chitatel' schitaet etot tekst napisannnym po-angliiski $i$ poetomu vryadli poimet etu frazu [18. Clearly, such a venture has a chance if and only if those languages are not completely different, but merely dialects of one language. Similarly, infinitessimally small changes to the interaction matrix should not have any significant ramifications, while on the other hand, a radically different matrix structure should lead to completely different folding behavior. Using the terminology of frozen and target phases, we can ask if the chain designed with some matrix $B_{i j}^{p}$ will freeze to target state when governed by another matrix $B_{i j}$ ? In other words, if we want to get the target phase, how accurate should we be in choosing matrices $B_{i j}^{p}$ and $B_{i j}$ ? Another interesting aspect of the question is which properties of $B_{i j}^{p}$ and $B_{i j}$ matrices are important, that is to which of them the chain behavior is sensitive? And what measure do we use to define the proximity of interaction matrices?

Previous treatments [17, I] have addressed certain aspects of these questions. However, they differ from the present work in that we model the effect of evolutionary optimization and the nullification of this by errors in the interaction potentials, whereas Refs. [17,1] examine the stability of glassy (not evolutionarily optimized) conformations with respect to errors.

\section{THE MODEL}

We start from a heteropolymer chain Hamiltonian in which interactions are described in terms of the energy of interaction of species

$$
\mathcal{H}=\sum_{I, J}^{N} B_{s_{I} s_{J}} \delta\left(\mathbf{r}_{I}-\mathbf{r}_{J}\right)
$$

where $B_{i j}$ is the interaction energy between monomer species $i$ and $j(i, j \in\{1 \ldots q\}), s_{I}$ is the species of monomer at position $I$ along the chain, $N$ is the number of monomers, and 
$\mathbf{r}_{I}$ is the position of monomer $I$. We use the convention that lower case roman letters label species space, upper case roman letters label monomer number along the chain, and lower case greek letters label replicas.

We do not explicitly include in the Hamiltonian (11) anything leading to the overall collapse of the chain. We do imply, however, the existence of some strong compressing factor, such as overall homopolymeric-type poor solvent effect (expressed with $\mathcal{H}^{\prime}=B \rho^{2}+C \rho^{3}$ with species independent $B$ and $C$ and strongly negative $B$ ) or box-like external field such that the polymer is always in a globular conformation. The particular choice of compressing factor is known to be unimportant [23] provided that the chain is long enough; we do not discuss here any finite size effects related to the surface of the globule even though these might be important for real proteins. Furthermore, we stress that this is of vital importance for the entire approach that the chain is maintained in the globular compact state (compare with Ref. [19], where the design scheme failed to work just because the requirement of overall collapsed state was relaxed).

Since the heteropolymer sequence does not change during folding, we immediately encounter the technical problem that sequences are a quenched quantity and thus we average the free energy over all sequences (with a particular weighting due to design) rather than the partition function. This leads directly to the replica approach. The details of the corresponding calculation are similar to what is presented elsewhere [16]. Here we briefly outline the main steps. The replicated partition function can be symbolically written as

$$
\left\langle Z^{n}\right\rangle=\sum_{\text {sequence }} \mathcal{P}_{\text {sequence }} \sum_{\{\text {conformations }\}} \exp \left[-\sum_{\alpha=1}^{n} \mathcal{H}\left(\text { sequence, conformation }{ }_{\alpha}\right) / T\right] \text {, }
$$

where we explicitly mention the dependence of the Hamiltonian (11) on both sequence, which is the same for all replicas $\alpha \in 1 \ldots n$, and conformation, which is potentially different for different replicas. Probability distribution over the set of sequences, $\mathcal{P}_{\text {sequence }}$ is defined by the preparation process and thus in our case can be written as

$$
\mathcal{P}_{\text {sequence }} \sim\left[p_{s_{1}} \cdot p_{s_{2}} \cdot \ldots \cdot p_{s_{N}}\right] \times
$$




$$
\times \sum_{\text {target conformation }} \exp \left[\mathcal{H}^{p} \text { (sequence, target conformation) } / T_{p}\right],
$$

where we drop the normalization factor. In the equation (3), $p_{s}$ is the probability of appearance of the monomer species $s$ (which is normally controlled by the chemical potentials of components in the monomer soup surrounding the preparation bath), $\mathcal{H}^{p}$ is Hamiltonian of the form (II) except with the "preparation" matrix $\widehat{B}^{p}$ instead of $\widehat{B}$ which controls folding through equation (2). Accordingly, $T_{p}$ is the temperature at which preparation process is performed.

We stress that our approach is not restricted to any particular target conformation. By contrast, we do average over all possible (compact) target conformations (see equation (3)), and thus our scheme picks up not just the good sequences, but the pairs "target conformation - sequence which is good for this target conformation," where both terms are well adjusted to each other (see also the discussion in Ref. [19]). This is a good match for Imprinting, since we assume that some external field chooses sequence-conformation pairs based upon matching with the field [20]. Indeed, this may be analogous to protein evolution, in which nature chooses sequence-conformation pairs not for any specific nature of the conformation or sequence but for its functionality; this can be viewed in physical terms as some external field affecting the selection of sequence and conformation [20].

\section{FREE ENERGY OF THE MODEL}

Inspection of the equations (2, 3) indicates that we can formally express the weight corresponding to the design process as an additional replica labeled 0 [14,16,21]:

$$
\left\langle Z^{n}\right\rangle=\sum_{\text {sequence }} \prod_{I=1}^{N} p_{s_{I}} \sum_{\{\text {conformations }\}} \exp \left[\sum_{\alpha=0}^{n} \sum_{I \neq J=1}^{N} B_{s_{I}, s_{J}}^{\alpha} \delta\left(\mathbf{r}_{I}^{\alpha}-\mathbf{r}_{J}^{\alpha}\right) / T_{\alpha}\right],
$$

where $B_{i j}^{\alpha=0} \equiv \widehat{B}^{p}$ is the matrix which expresses the interactions used for the chain preparation (i.e. replica $\alpha=0$ ) and $B_{i j}^{\alpha>0} \equiv B_{i j}$ is the interaction matrix which governs folding or renaturation. Hereafter, conformations are given in terms of position vectors $\mathbf{r}_{I}^{\alpha}$ for each monomer number $I$ and each replica $\alpha$. By the sum over conformations we mean the sum 
in which the condition of chain connectivity is strictly obeyed (technically this can be done either in continuous form as Edwards [22] or in discrete form like Lifshits [23]).

To facilitate averaging over the sequences, we define the densities

$$
\rho_{i}^{\alpha}(\mathbf{R})=\sum_{I}^{N} \delta\left(s_{I}, i\right) \delta\left(\mathbf{r}_{I}^{\alpha}-\mathbf{R}\right),
$$

then rewrite the exponent in equation (雨) as

$$
\sum_{I \neq J=1}^{N} \frac{B_{s_{I}, s_{J}}^{\alpha}}{T_{\alpha}} \delta\left(\mathbf{r}_{I}^{\alpha}-\mathbf{r}_{J}^{\alpha}\right)=\int d \mathbf{R}_{1} d \mathbf{R}_{2} \sum_{i, j}^{q} \rho_{i}^{\alpha}\left(\mathbf{R}_{1}\right) \frac{B_{i, j}^{\alpha}}{T_{\alpha}} \delta\left(\mathbf{R}_{1}-\mathbf{R}_{2}\right) \rho_{j}^{\alpha}\left(\mathbf{R}_{2}\right)
$$

and perform a Hubbard-Stratonovich transformation on the quantity $\rho_{i}^{\alpha}(\mathbf{R})$, thus introducing the conjugate field $\phi_{i}^{\alpha}(\mathbf{R})$. We average over the sequence and truncate the resulting exponent to $\mathcal{O}\left(\phi^{2}\right)$, which yields (see the details in Ref. [16]):

$$
\begin{aligned}
& \left\langle Z^{n}\right\rangle=\sum_{\text {conformations }} \int \mathcal{D}\{\phi(\mathbf{R})\} \exp \left\{\int d \mathbf{R} \sum_{\alpha=0}^{n} \sum_{i}\left[p_{i} \rho^{\alpha}(\mathbf{R}) \phi_{i}^{\alpha}(\mathbf{R})\right]+\right. \\
& \left.\int d \mathbf{R}_{1} d \mathbf{R}_{2} \sum_{\alpha, \beta=0}^{n} \sum_{i j}\left[\frac{1}{4}\left(\frac{B_{i j}^{\alpha}}{T_{\alpha}}\right)^{-1} \delta^{\alpha \beta} \delta\left(\mathbf{R}_{1}, \mathbf{R}_{2}\right)+\frac{1}{2} \Delta_{i j} Q^{\alpha \beta}\left(\mathbf{R}_{1}, \mathbf{R}_{2}\right)\right] \phi_{i}^{\alpha}\left(\mathbf{R}_{1}\right) \phi_{j}^{\beta}\left(\mathbf{R}_{2}\right)\right\},
\end{aligned}
$$

where we define the overall density $\rho_{\alpha}(\mathbf{R})=\sum_{I}^{N} \delta\left(\mathbf{r}_{\mathbf{I}}^{\alpha}-\mathbf{R}\right)=\sum_{i=1}^{q} \rho_{i}^{\alpha}(\mathbf{R})$ and the replica overlap order parameter $Q_{\alpha \beta}\left(\mathbf{R}_{1}, \mathbf{R}_{2}\right)=\sum_{I}^{N} \delta\left(\mathbf{r}_{\mathbf{I}}^{\alpha}-\mathbf{R}_{1}\right) \delta\left(\mathbf{r}_{\mathbf{I}}^{\beta}-\mathbf{R}_{2}\right)$. Since the density is a single replica quantity and we assume the chain as a whole is compressed, that is, density is constant throughout the globule, we simply take $\rho_{\alpha}(\mathbf{R}) \equiv \rho$. Furthermore, using a variational argument, it was shown [9:14] that freezing occurs down to microscopic length scales, thus allowing to take $Q_{\alpha \beta}\left(\mathbf{R}_{1}, \mathbf{R}_{2}\right)=\rho q_{\alpha \beta} \delta\left(\mathbf{R}_{1}-\mathbf{R}_{2}\right)$, where the form of the conformation correlator $q_{\alpha \beta}$ is found to be that of a Parisi matrix with one step symmetry breaking, with either complete overlap $\left(q^{\alpha \beta}=1\right)$ or no overlap $\left(q^{\alpha \beta}=0\right)$. (This directly corresponds with the Random Energy Model [24] introduced directly in previous heteropolymer models [5].) This facilitates Gaussian integration over the $\phi$ fields. To write the result in even simpler form, we can also include a conformation-independent constant by the transformation

$$
\begin{aligned}
& \phi_{i}^{\alpha} \rightarrow 2 \sum_{j}\left(\rho \widehat{B}^{\alpha} / T_{\alpha}\right)_{i j}^{1 / 2} \phi_{j}^{\alpha} \text { to get } \\
&\left\langle Z^{n}\right\rangle=\sum_{\text {conformations }}\left[\int d \{ \phi \} \operatorname { e x p } \left\{\sum_{\alpha=0}^{n} \sum_{i j}\left[\left(2 \rho \widehat{B}^{\alpha} / T_{\alpha}\right)_{i j}^{1 / 2} p_{i} \phi_{j}^{\alpha}\right]+\right.\right.
\end{aligned}
$$




$$
\left.\left.\sum_{\alpha, \beta=0}^{n} \sum_{i j}\left[\delta_{i j} \delta^{\alpha \beta}+2\left(\left(\widehat{B}^{\alpha} / T_{\alpha}\right)^{1 / 2} \widehat{\Delta}\left(\widehat{B}^{\beta} / T_{\beta}\right)^{1 / 2}\right)_{i j} \rho q^{\alpha \beta}\right] \phi_{i}^{\alpha} \phi_{j}^{\beta}\right\}\right]^{N}
$$

where we use a hat to indicate that the object is matrix in species space (i.e. $\widehat{A}=A_{i j}$ ). We evaluate this Gaussian integral, yielding the free energy

$$
\left\langle Z^{n}\right\rangle=\sum_{\{\text {conformations }\}} \exp [E(q)]
$$

where the effective energy of the $n$ replica system is given by

$$
\begin{aligned}
\frac{E(q)}{N}= & \frac{1}{2} \ln \operatorname{det}\left[\widehat{I} \delta^{\alpha \beta}+2 \rho\left(\widehat{B}^{\alpha} / T_{\alpha}\right)^{1 / 2} q^{\alpha \beta} \widehat{\Delta}\left(\widehat{B}^{\beta} / T_{\beta}\right)^{1 / 2}\right]+ \\
& \frac{1}{\rho} \sum_{\alpha \beta}\left\langle\vec{\rho}\left|\left(\widehat{B}^{\alpha} / T_{\alpha}\right)^{1 / 2}\left[\widehat{I}^{\alpha \beta}+2 \rho\left(\widehat{B}^{\alpha} / T_{\alpha}\right)^{1 / 2} q^{\alpha \beta} \widehat{\Delta}\left(\widehat{B}^{\beta} / T_{\beta}\right)^{1 / 2}\right]^{-1}\left(\widehat{B}^{\beta} / T_{\beta}\right)^{1 / 2}\right| \vec{\rho}\right\rangle,
\end{aligned}
$$

$\langle|\cdots|\rangle$ denotes the scalar product over species space, the determinant in the first term is over species and replica space, and the vector $\vec{\rho}$ is given by $\vec{\rho}_{i}^{\alpha}=p_{i} \rho$. Note that the only remaining dependence on conformations come through conformational correlators $q^{\alpha \beta}$. Given the particular structure of $q^{\alpha \beta}$, effective energy (10) can be expressed directly in terms of the number of replicas which overlap with the target group $y$ and the size of a group $x$ for the remaining $n-y$ replicas divided into $(n-y) / x$ groups. Thus, we can simplify the expression for effective energy (10) by removing replica dimensionalities, as is performed in Appendix A. This also allows one to write the entropy of the macrostate with given $x$ and $y$, as it is associated simply with grouping of replicas $S=N s[y+(n-y)(x-1) / x]$. identical conformation down to microscopic scale related to the volume $v$, there is an entropy loss of $s \equiv \ln \left(a^{3} / v\right)$ per monomer, where $a$ is the distance between monomers and $v$ is the excluded volume [8,9]. This allows conversion from the sum over conformations to a functional integral over $Q^{\alpha \beta}\left(\mathbf{R}_{1}, \mathbf{R}_{2}\right)$, and even further, to conventional integral over $x$ and $y$, which, in the mean field approximation, can be further simplified to optimization of the effective $n$-replica free energy

$$
\begin{aligned}
\frac{F(x, y)}{N} & =\frac{n-y}{2 x}\left\{\ln \operatorname{det}[\widehat{I}+2 x \widehat{\Delta} \widehat{B} / T]+\left\langle\vec{p}\left|2 x \widehat{B}^{1} / T[\widehat{I}+2 x \widehat{\Delta} \widehat{B} / T]^{-1}\right| \vec{p}\right\rangle\right\} \\
& +\frac{1}{2} \ln \operatorname{det}\left[\widehat{I}+2 \widehat{\Delta} \widehat{B}^{p} / T_{p}+2 y \widehat{\Delta} \widehat{B} / T\right]
\end{aligned}
$$




$$
\begin{aligned}
& +\left\langle\vec{p}\left|\left[\widehat{B}^{p} / T_{p}+y \widehat{B} / T\right]\left[\widehat{I}+2 \widehat{\Delta} \widehat{B}^{p} / T_{p}+2 y \widehat{\Delta} \widehat{B} / T\right]^{-1}\right| \vec{p}\right\rangle \\
& -s[y+(n-y)(x-1) / x]
\end{aligned}
$$

\section{ANALYSIS OF THE FREE ENERGY AND PHASE DIAGRAM}

The expression (11) is rather similar to what we had in Ref. [16] while considering the model with identical interactions for design and folding and, of course, it is exactly reduced to the corresponding equation of that work [1] 6 when $\widehat{B}=\widehat{B}^{p}$. Furthermore, this expression implies the same structure of phase diagram, with the same three globular phases: random, frozen, and target. (We remind the reader, that overall collapse of the chain is the necessary pre-condition of our approach, and thus globule-to-coil phase transition falls outside of the framework of the present study). To see the structure of phase diagram, we first look at the allowed variations of the order parameters $x$ and $y$.

For simplicity, we consider here only small $s$ regime. In this case, freezing transitions, which are the main topic of our interest here, occur when $B$ is (in a reasonable sense) also small. Indeed, freezing phase transitions result physically from the competition between energetic and entropic parts of free energy (11), where energetic part favors gathering of replicas into groups while entropic part favors diversity of replicas. For energy to be competitive to an entropy when $s$ is small, $B$ must be small as well. This allows one to simplify equation (11) truncating it to quadratic order in $B$.

As $y$ is the number of replicas whose conformation coincides with the target conformation, this value must be in between of 0 and $n$. What is relevant in replica approach is $n \rightarrow 0$ limit, and, moreover, only the terms which are linear in $n$ are to be considered (because higher order terms disappear in the main equation $\left.\langle\ln Z\rangle=\lim _{n \rightarrow 0}\left(\left\langle Z^{n}\right\rangle-1\right) / n\right)$. Accordingly, since $0 \leq y \leq n$, we must linearize the free energy in $y$ as well [14, 116]. This leads to further simplification of (11):

$$
F=\operatorname{Tr}\left[(n-y)\left\{\widehat{\Delta} \widehat{B} / T-x \widehat{\Delta} \widehat{B} \widehat{\Delta} \widehat{B} / T^{2}+\widehat{P} \widehat{B} / T-2 x \widehat{P} \widehat{B} \widehat{\Delta} \widehat{B} / T^{2}\right\}\right.
$$




$$
\begin{aligned}
& +\widehat{\Delta} \widehat{B}^{p} / T_{p}+y \widehat{\Delta} \widehat{B} / T-2 y \widehat{\Delta} \widehat{B}^{p} \widehat{\Delta} \widehat{B} / T T_{p}-\widehat{\Delta} \widehat{B}^{p} \widehat{\Delta} \widehat{B}^{p} / T_{p}^{2} \\
& \left.+\widehat{P} \widehat{B}^{p} / T^{p}+y \widehat{P} \widehat{B} / T-2\left(\widehat{P} \widehat{B}^{p} \widehat{\Delta} \widehat{B}^{p} / T_{p}^{2}+y \widehat{P} \widehat{B} \widehat{\Delta} \widehat{B}^{p} / T T_{p}+y \widehat{P} \widehat{B}^{p} \widehat{\Delta} \widehat{B} / T T_{p}\right)\right] \\
& -T N s[y+(n-y)(x-1) / x]
\end{aligned}
$$

where $P_{i j} \equiv p_{i} p_{j}$.

While $y$ describes breaking of the symmetry between $n$ replicas due to their attraction to the target replica labeled $0, x$ describes spontaneous symmetry breaking. When we have integer number of replicas, $n$, clearly, $1 \leq x \leq n$ : $x$ cannot be smaller than unity, because it is the number of replicas in the group. When $n \rightarrow 0$, the logic about the number of replicas in the group is not applicable any more, but it is natural to think that formal inequalities for $x$ just simply flip signs: $n \leq x \leq 1$. With this in mind, we optimize free energy (12) with respect to $x$ yielding the equation which determines $x$ :

$$
s=\frac{x^{2}}{T^{2}} \operatorname{Tr}[\widehat{\Delta} \widehat{B} \widehat{\Delta} \widehat{B}+2 \widehat{P} \widehat{B} \widehat{\Delta} \widehat{B}]
$$

Note, that this equation does not involve either $T_{p}$ or $\widehat{B}^{p}$ and thus it does not depend on preparation process. This has clear physical meaning. Namely, this reflects the behavior similar to that of REM, because the designed sequence behaves precisely as a random one in all the conformations except for the target conformation.

At this point, it is useful to introduce the following matrix "cumulants":

$$
\begin{aligned}
\langle A\rangle_{c} & \equiv \sum_{i, j} p_{i} p_{j} A_{i j}=\operatorname{Tr}(\widehat{P} \widehat{A}) \\
\langle A B\rangle_{c} & \equiv \sum_{i, j} p_{i} p_{j} A_{i j} B_{i j}-\langle A\rangle_{c}\langle B\rangle_{c}=\operatorname{Tr}(\widehat{\Delta} \widehat{A} \widehat{\Delta} \widehat{B}+2 \widehat{P} \widehat{A} \widehat{\Delta} \widehat{B})
\end{aligned}
$$

where $\widehat{A}$ and $\widehat{B}$ are arbitrary matrices.

From the above, we can easily find the equation for the freezing temperature for random sequences. Indeed, freezing occurs when replicas start to group, thus spontaneously breaking the permutation symmetry. This happens when $x=1$. Therefore, freezing temperature is given via the relation

$$
T_{f}^{2}=\langle B B\rangle_{c} / s
$$


In other words, the freezing temperature is given by the variance of the renaturation interaction matrix [10]. Note that this is a transition to a unique ground state which is not necessarily (and most likely not) the target conformation: we call this phase the frozen phase and we call the high temperature disordered phase in which there is no form of freezing, i.e. many conformations dominate equilibrium, the random phase.

To examine freezing to the target conformation, we must examine the conditions at which $y>0$. Since $y$ varies from 0 to $n$, what has physical meaning in the $n \rightarrow 0$ limit is only the linear in $y$ term of free energy. Therefore, free energy optimum corresponds to either $y=0$ (non-target phase), or to $y=n$ (target phase). To find the corresponding critical temperature, we must examine the slope of the free energy at the point $y=0$ to determine whether $y=0$ or $y=n$ is the stable solution [16]. The condition "slope" $=0$ yields the relationship:

$$
\begin{aligned}
s & =\operatorname{Tr}\left[x\left(\frac{\widehat{\Delta} \widehat{B}}{T} \frac{\widehat{\Delta} \widehat{B}^{p}}{T_{p}}+2 \frac{\widehat{P} \widehat{B}}{T} \frac{\widehat{\Delta} \widehat{B}^{p}}{T_{p}}+\frac{\widehat{\Delta} \widehat{B}^{p}}{T_{p}} \frac{\widehat{\Delta} \widehat{B}}{T}+2 \frac{\widehat{P} \widehat{B}^{p}}{T_{p}} \frac{\widehat{\Delta} \widehat{B}}{T}\right)-\frac{x^{2}}{T^{2}}(\widehat{\Delta} \widehat{B} \widehat{\Delta} \widehat{B}+2 \widehat{P} \widehat{B} \widehat{\Delta} \widehat{B})\right] \\
& =\frac{2 x}{T_{p} T}\left\langle B^{p} B\right\rangle_{c}-\frac{x^{2}}{T^{2}}\langle B B\rangle_{c}
\end{aligned}
$$

This equation defines the phase boundary of the target phase, in which the system freezes to the target conformation.

We combine eqns $(13-16)$ to get the boundary of the target phase (i.e. the preparation temperature $T_{p}$ which separates the target phase from the random and frozen globule phases). To write the result, it is convenient to define formally the value of $T_{p f}$ according to the equation

$$
T_{p f}^{2}=\left\langle B^{p} B^{p}\right\rangle_{c} / s
$$

similar to eq (15) except it includes preparation matrix $\widehat{B}^{p}$ instead of $\widehat{B}$. Physically, $T_{p f}$ is the temperature point at which random heteropolymer would undergo freezing transition provided its conformations are governed by $\widehat{B}^{p}$ interaction matrix; $T_{p f}$ gives a natural scale for $T_{p}$ : 


$$
\frac{T_{p}^{(c r)}}{T_{p f}}=\left\{\begin{array}{ll}
g \frac{2 T / T_{f}}{\left(1+T^{2} / T_{f}^{2}\right)} & \text { for } T \geq T_{f} \\
g & \text { for } T \leq T_{f}
\end{array} ;\right.
$$

as far as the small $s$ limit is concerned, this can be also rewritten in terms of $T_{\mathrm{tar}}$, acting temperature at which random-to-target phase transition occurs:

$$
\frac{T_{\mathrm{tar}}}{T_{f}}=1+\left[1-\frac{T_{p}}{g T_{p f}}\right]^{1 / 2} .
$$

This is the previously obtained result for the transition to the target phase [16], except with the inclusion of a factor $g$, which is defined as

$$
g \equiv\left\langle B^{p} B\right\rangle_{c} / \sqrt{\langle B B\rangle_{c}\left\langle B^{p} B^{p}\right\rangle_{c}} .
$$

To understand the meaning of $g$, it must be noted first of all that $g$ can be treated as scalar product, $g=\cos \theta$, and thus $-1 \leq g \leq 1$. This factor gives the degree of correlation between the elements of the two matrices, $\widehat{B}$ and $\widehat{B}^{p}$. If the two matrices are the same (i.e. completely correlated) $g=1$. The region $0<g<1$ corresponds to somewhat lesser, but still positive, degree of correlation; $g=0$ means that matrices are statistically independent; $-1<g<0$ corresponds to some anti-correlation; finally, $g=-1$ means absolute anticorrelation (each pair of monomers which is supposed to be attractive in $\widehat{B}$, is repulsive in $\widehat{B}^{p}$, and vice versa, etc). To see this, it is helpful to note, that the definition of matrix cumulants has the property that $\left\langle B^{1} B^{2}\right\rangle$, where $\widehat{B}^{1}$ and $\widehat{B}^{2}$ are both either $\widehat{B}$ or $\widehat{B}^{p}$, does not change upon adding a constant to all matrix elements (this can be easily proven given that $\sum_{i} p_{i}=1$ ); this allows to define $b_{i j}^{x}=B_{i j}^{x}-\left\langle B^{x}\right\rangle_{c}$ and rewrite eq (20) as $g=\left\langle b^{p} b\right\rangle_{c} / \sqrt{\langle b b\rangle_{c}\left\langle b^{p} b^{p}\right\rangle_{c}}$. It is now seen, that, for example, $g=-1$ corresponds to $b_{i j}=-b_{i j}^{p}$.

According to eq (18), the value $T_{p}^{(c r)}$ is proportional to $g$. At $g=1$, we recover the result for $\widehat{B}^{p}=\widehat{B}[\mathbb{1 6}]$. Appearance of positive, but not absolute correlation $(0<g<1)$ has simple graphical meaning on the phase diagram, Fig. 1 - it leads to affine deformation of the boundary of target phase region on the phase diagram. At $g=0$, the target region disappears, and it does not exist at $g<0$. This is clear, because when matrices are anticorrelated, "design" does not help, but rather destroys the chances of polymer to fold into 
desirable conformation. Thus, the correlation between matrices, given by the factor $g$, is the measure of the proximity between interaction matrices.

\section{DISCUSSION}

By performing explicit calculations for the freezing transition of heteropolymers with different matrices for design and renaturation, we have found three phases: random, in which many conformations dominate equilibrium; frozen, where the polymer freezes to a single conformation other than the target conformation; and target, in which the polymer freezes to the target conformation. In the flexible chain limit, for the case where the design and renaturation matrices are different, the effective critical selective temperature for renaturation to the target phase becomes modified by a factor from the normalized correlation between the matrices $\left(T_{p}^{(c r)} \rightarrow T_{p}^{(c r)} g\right)$. For complete correlation, $g=1$. For differences in the design and renaturation matrix $(g<1)$, special measures must be undertaken in order to keep the system in the target phase; otherwise, there is no possibility to obtain renaturation to the correct target conformation.

To understand better the meaning of the result obtained, consider that proteins have been indeed "designed" according to one of the theoretical models [ [0 [5]. This means, that they were prepared under the interactions $\widehat{B}^{p}$ at some temperature $T_{p}<T_{p f}$, and now they "work" at some other temperature $T$, such that $T_{p f}<T<T_{\operatorname{tar}}$. It is worth stressing, that

their "work" is governed by their natural interactions, that is, by the same matrix $\widehat{B}^{p}$ as was supposedly used for "design." We take now some other artificial matrix $\widehat{B}$ and try (for example, by means of computer simulation) to recover the correct renaturation. In terms of our phase diagram, Fig. 1, correct renaturation occurs when and only when the system remains in the target phase. This is illustrated graphically in Fig. 1: the "Natural" phase diagram is presented there with solid lines, and real proteins are supposedly represented by the point within target phase region. Phase diagrams of a couple of artificial systems, with sequences designed by natural matrix $\widehat{B}^{p}$ and conformations governed by mistaken matrices 
$\widehat{B}$, are shown with dashed lines; a greater degree of errors in the potentials push the phase boundary to the left, determined by the value of the factor $g$ (defined by (20)). In the example illustrated in the figure, the representative point for matrices with $g>0.95$ remain within the target phase region, while those with $g<0.95$ do not possess this property. In the first case, correct renaturation can be recovered, in the second case this is impossible. We conclude, that correct renaturation is possible when the degree of correlation is sufficient between $\widehat{B}^{p}$ and $\widehat{B}$, namely, when

$$
g>g^{*} \quad(\text { the condition for correct renaturation) }
$$

where $g^{*}$ is defined from the condition that the boundary $T_{\operatorname{tar}}$, given by the equation (19), goes through the given point $\left(T_{p}, T\right)$ :

$$
g^{*}=\frac{T_{p}}{T_{p f}}+\left[\frac{T}{T_{f}}-1\right]^{2} .
$$

This can be also instructively rewritten as

$$
g^{*}=1+\frac{T_{p}^{(c r)}-T_{p}}{T_{p f}} .
$$

Note, that the ratio $T_{p} / T_{p f}$ can serve as a measure of degree of selection of sequences: smaller values of $T_{p} / T_{p f}$ correspond to stronger selection of sequences. At the same time, $\left[T_{p}^{(c r)}-T_{p}\right] / T_{p f}$ measures the degree of necessary selection at the given actual temperature, $T$ (because $T_{p}^{(c r)}$ depends on $T$, equation (18)). We conclude, that minimal "correctness" of interaction matrix, $g^{*}$, is defined by the degree of selection, or optimization, of the set of real sequences: the better they have been optimized, the more stable is their renaturation with respect to the mistakes in interactions.

Speaking about the numbers involved in the problem, we have to stress that the information available is by far insufficient to make any solid statements. To get some rough idea, we can proceed in the following way. When extracting a matrix of species-species energies for proteins from the statistics of protein data bank, such as the Miyazawa and Jernigan (MJ) matrix [25], what one obtains is actually $B_{i j}^{\mathrm{MJ}}=B_{i j}^{p} / T_{p}$ (see Ref. [27] and Appendix B). 
Since from equation (15) we have, $\left\langle B_{i j}^{2}\right\rangle_{c}^{1 / 2}=s^{1 / 2} T_{f}$, then the variance of the MJ matrix yields $\left\langle\left(B_{i j}^{\mathrm{MJ}}\right)^{2}\right\rangle_{c}^{1 / 2}=s^{1 / 2} T_{p f} / T_{p}$; therefore, with the knowledge of the MJ matrix $\left(\left\langle\left(B_{i j}^{\mathrm{MJ}}\right)^{2}\right\rangle_{c} \approx 2.0\right)$ and the flexibility of proteins $s(s \approx 1.6)$, we also arrive at $T_{p} / T_{p f} \approx 0.9$. It is also independently hypothesized [26] that the ratio of the "folding" to the "glass" temperature should be about 1.6. Without going into the arguments of the work Ref. [26], we can, quite arbitrarily, identify "folding" temperature with $T_{\text {tar }}$ and "glass" temperature with $T_{p f}$; by doing so, we obtain $T_{\operatorname{tar}} / T_{f} \approx 1.6$, which, in view of the equation (18), yields a similar estimate for the degree of optimization $T_{p} / T_{p f} \approx 0.9$. We conclude, that a conservative estimate of $g^{*}$ is likely to be about $g^{*} \approx 0.95$ : correct recovery of the native state require $g>0.95$, if $g<0.95$ chances of correct renaturation are slim.

Yet another, though purely algebraical, aspect of the problem is which types and values of errors in determination of interactions lead to a particular value of $g$ factor, such as, for instance, 0.95 mentioned above. We first of all note that neither additive $\left(B_{i j}=B_{i j}^{p}+B_{0}\right)$ nor multiplicative $\left(B_{i j}=\beta B_{i j}^{p}\right)$ systematic errors do not contribute at all, $g=1$ in both cases (as well as in the "combined" case $B_{i j}=\beta B_{i j}^{p}+B_{0}$ ). This is clear physically because these kinds of errors contribute to homopolymer terms only and do not affect selectivity of interactions of monomers to one another. On a more formal level, additive constants does not change second moments of matrices defined according to eq (14), and multiplicative constant obviously does not affect the value of $g(20)$. To get an idea about random mistakes, we examine the case where the renaturation matrix is the design matrix with some normally distributed noise $\eta_{i j}: B_{i j}=B_{i j}^{p}\left(1+\eta_{i j}\right)$, where $\mathcal{P}\left(\eta_{i j}\right) \propto \exp \left[-\eta_{i j}^{2} / \sigma^{2}\right]$. We can average the $g$ factor over the noise to get

$$
\bar{g}=\frac{\left\langle B_{i j}^{p}\left[B_{i j}^{p}\left(1+\eta_{i j}\right)\right]\right\rangle}{\left[\left\langle\left[B_{i j}^{p}\left(1+\eta_{i j}\right)\right]^{2}\right\rangle\right]^{1 / 2}}=\left(1+\sigma^{2}\right)^{-1 / 2}
$$

This gives $\sigma \approx 10 \%$. More complicated random, systematic and mixed errors may be interesting to model and this can be easily accomplished within this formalism but the results are dependent of the specific nature of these errors and are therefore not within the 
more general scope of this paper.

It is worth making very clear that this error limit is independent of the length of the polymer. Previous calculations [1] have made estimates which are directly based upon $N$ (ie. the error must be small compared with $1 / \sqrt{N}$ ). This reflects fundamental difference of our approach from that of the Ref. [1]; even more, this reflects the difference between questions studied. In the work Ref. [1], calculations were performed for random heteropolymers, neither any kind of design nor the principle of minimal frustration was imposed; accordingly, the question studied was in fact about the possibility to reconstruct the randomly chosen conformation of frozen globule phase. In the ensemble of random sequences, the ones with very stable ground state are very (exponentially) rare, thus, it is not surprising that these ground states are typically very unstable with respect to error-based renaturation, especially for long chains. By contrast, our treatment is a comparison between the types of freezing (to the target or some random conformation). This is therefore independent of the length of the polymer chain and essentially of a different nature than that of Ref. [1]. Furthermore, within our formalism, the transition in $y$, the number of replicas in the target group, is first order; therefore, in the framework of our approach one cannot discuss the "degree" of renaturation in terms of a given percentage of correct contacts: in thermodynamic equilibrium, and in very long chain (thermodynamic limit) either there is renaturation to the target conformation or folding to some entirely different conformation.

Within the framework of our formalism, the Independent Interaction Model [8] can be recovered by addressing the limit $q \rightarrow N$ and assuming that $B$ is a normally distributed matrix; in this case eq (15) agrees with the results of more direct calculations of this model 88. The error limits in this approximation are derived in exactly the same manner as (24). This is not surprising as, in fact, the validity of the approximation of taking the free energy to $\mathcal{O}\left(B^{2}\right)$ in eq (12) is similar to that of the Independent Interaction Model [16]: we assume that the effective flexibility $s$ of the polymer is small. However, our treatment allows corrections to this approximation to be systematically derived.

In conclusion, starting from the most general Hamiltonian involving short range binary 
heteropolymeric interactions, we have derived what measure is used to compare differences in interaction potentials and the limits in which renaturability to the target conformation is still allowed. Simple estimates of normally distributed error indicates that even conservative estimates leave room for $10 \%$ error in potentials. Using our formalism, one can make a more informed estimate based upon more precise knowledge of the form of errors involved, i.e. the correlations of errors in the matrix.

\section{ACKNOWLEDGEMENTS}

The work was supported by NSF (DMR 90-22933) and NEDO of Japan. VSP acknowledges the support of an NSF Fellowship. AYG acknowledges the support of Kao Fellowship. 


\section{APPENDIX A: SIMPLIFICATION OF EQUATION (7)}

We will a slightly different notation from the rest of the paper to facilitate calculations: we eliminate indices and simply give the dimensionality of the operators explicitly, eg. we label $\Delta_{i j}$ as $\widehat{\Delta}^{(q)}$ since it is a $q \times q$ dimensional matrix.

We perform the simplification of the elimination of replicas though several steps:

1. $\hat{q}$ is of well-known one-step replica symmetry breaking shape, with one distinct group of $y+1$ replicas and $(n-y) / x$ groups of $x$ replicas each.

2. $\widehat{M}=\widehat{I}+2 \rho \widehat{q} \otimes \widehat{\Delta} \widehat{B}$ can be viewed as $(n+1) \times(n+1)$ block matrix in replica space, with each matrix element being $q \times q$ matrix in species space. This block matrix is of the same structure as $\widehat{q}$, with one $(y+1) \times(y+1)$ super-block and $(n-y) / x$ of $x \times x$ super-blocks.

3. The determinant in the first term in free energy is decomposed into the product of determinants of super-blocks.

4. Vector $\vec{\rho}$ is composed of $n+1$ "blocks" $p_{i}$, thus making the second term in free energy the sum of independent contributions from the groups of replicas. Along with previous, this means that different groups of replicas do hot interact and this is why they contribute independently to the free energy.

5. Effective replica energy $E$ is now presented in the form

$$
\frac{E(x, y)}{N}=\epsilon_{y}+\frac{n-y}{x} \epsilon_{x}
$$

where $\epsilon_{y}$ and $\epsilon_{x}$ are the (independent) contributions from the corresponding groups of replicas. (Note, that replica entropy is also of the same form).

6. Both $\epsilon_{y}$ and $\epsilon_{x}$ have almost the same form as $E(\sqrt{10})$, except simpler matrix $\widehat{\tilde{q}}$, with all matrix elements 1 , appears instead of $\widehat{q}$ : 


$$
\begin{aligned}
\epsilon_{z} & =\frac{1}{2} \ln \operatorname{det}\left[\widehat{I}^{(z q)}+2 \rho \widehat{\tilde{q}}^{(z)} \otimes \widehat{\Delta}^{(q)} \widehat{B}^{(z q)}\right]+ \\
& +\frac{1}{\rho}\left\langle\vec{\rho}\left|\widehat{B}^{(z q)}\left[\widehat{I}^{(z q)}+2 \rho \widehat{\tilde{q}}^{(z)} \otimes \widehat{\Delta}^{(q)} \widehat{B}^{(z q)}\right]^{-1}\right| \vec{\rho}\right\rangle,
\end{aligned}
$$

where $z$ is either $x$ or $y+1$, i.e., the number of replicas in the group.

7. To simplify first term (with determinant), we define rotation unitary operator

$$
\widehat{\mathcal{R}}_{\alpha \beta}^{(z)}=\frac{1}{\sqrt{z}} \exp \left[\frac{2 \pi i}{z}(\alpha-1)(\beta-1)\right] \quad 1 \leq \alpha, \beta \leq z .
$$

It is easy to check that this operator transforms $\widehat{\tilde{q}}^{(z)}$ into diagonal form, where one diagonal matrix element is 1 , while all others are 0 :

$$
\widehat{\mathcal{R}}^{(z)} \widehat{\widetilde{q}}^{(z)}\left(\widehat{\mathcal{R}}^{(z)}\right)^{-1}=\widehat{\lambda}^{(z)}, \quad \text { where } \quad \widehat{\lambda}_{\alpha \beta}=z \delta_{\alpha 1} \delta_{1 \beta} .
$$

We define also $\widehat{\mathcal{R}}^{(z q)}=\widehat{I}^{(q)} \otimes \widehat{\mathcal{R}}^{(z)}$ and note that the determinant is not changed upon rotation. We write

$$
\begin{aligned}
& \operatorname{det}\left[\widehat{I}^{(z q)}+2 \rho \widehat{\tilde{q}}^{(z)} \otimes \widehat{\Delta}^{(q)} \widehat{B}^{(z q)}\right]= \\
& =\operatorname{det}\left[\widehat{\mathcal{R}}^{(z q)}\right] \operatorname{det}\left[\widehat{I}^{(z q)}+2 \rho \widehat{\tilde{q}}^{(z)} \otimes \widehat{\Delta}^{(q)} \widehat{B}^{(z q)}\right] \operatorname{det}\left[\widehat{\mathcal{R}}^{(z q)}\right]^{-1} \\
& =\operatorname{det}\left[\widehat{I}^{(z q)}+2 \rho\left(\widehat{\mathcal{R}}^{(z q)}\right) \widehat{\tilde{q}}^{(z)} \otimes \widehat{\Delta}^{(q)} \widehat{B}^{(z q)}\left(\widehat{\mathcal{R}}^{(z q)}\right)^{-1}\right] \\
& =\operatorname{det}\left[\widehat{I}^{(z q)}+2 \rho\left(\widehat{\mathcal{R}}^{(z)}\right) \widehat{\tilde{q}}^{(z)}\left(\widehat{\mathcal{R}}^{(z)}\right)^{-1} \otimes \widehat{\Delta}^{(q)}\left(\widehat{\mathcal{R}}^{(z q)}\right) \widehat{B}^{(z q)}\left(\widehat{\mathcal{R}}^{(z q)}\right)^{-1}\right] \\
& =\operatorname{det}\left[\widehat{I}^{(z q)}+2 \rho \widehat{\lambda}^{(z)} \otimes \widehat{\Delta}^{(q)}\left(\widehat{\mathcal{R}}^{(z q)}\right) \widehat{B}^{(z q)}\left(\widehat{\mathcal{R}}^{(z q)}\right)^{-1}\right] \text {. }
\end{aligned}
$$

As $\widehat{B}^{(z q)}$ is diagonal in replica space, $\widehat{B}^{(z q)}=\widehat{B}_{\alpha}^{(q)} \delta_{\alpha \beta}$, we have

$$
\begin{aligned}
& \left(\left(\widehat{\mathcal{R}}^{(z q)}\right) \widehat{B}^{(z q)}\left(\widehat{\mathcal{R}}^{(z q)}\right)^{-1}\right)_{\alpha \beta}=\sum_{\gamma \delta} \widehat{\mathcal{R}}_{\alpha \gamma} \widehat{B}_{\gamma}^{(q)} \delta_{\gamma \delta}\left(\widehat{\mathcal{R}}^{(z q)}\right)_{\delta \beta}^{-1}= \\
= & \frac{1}{z} \sum_{\gamma} \exp \left[\frac{2 \pi i}{z}(\alpha-\beta)(\gamma-1)\right] \widehat{B}_{\gamma}^{(q)} .
\end{aligned}
$$

Taking into account the simple structure of $\widehat{\lambda}$ (A4), we arrive at

$$
\widehat{\lambda}^{(z)} \otimes \widehat{\Delta}^{(q)}\left(\widehat{\mathcal{R}}^{(z q)}\right) \widehat{B}^{(z q)}\left(\widehat{\mathcal{R}}^{(z q)}\right)^{-1}=\delta_{\alpha 1} \sum_{\gamma} \exp \left[\frac{2 \pi i}{z}(1-\beta)(\gamma-1)\right] \widehat{\Delta}^{(q)} \widehat{B}_{\gamma}^{(q)}
$$


8. First consider a non-target group of $z=x$ replicas. In this group, all the replicas are identical meaning that $\widehat{B}_{\gamma}^{(q)}=\widehat{B}^{(q)}$ does not depend on replica index $\gamma$. This yields

$$
\widehat{\lambda}^{(z)} \otimes \widehat{\Delta}^{(q)}\left(\widehat{\mathcal{R}}^{(z q)}\right) \widehat{B}^{(z q)}\left(\widehat{\mathcal{R}}^{(z q)}\right)^{-1}=x \delta_{\alpha 1} \delta_{1 \beta} \widehat{\Delta}^{(q)} \widehat{B}^{(q)}
$$

and thus

$$
\operatorname{det}\left[\widehat{I}^{(x q)}+2 \rho \widehat{\tilde{q}}^{(x)} \otimes \widehat{\Delta}^{(q)} \widehat{B}^{(x q)}\right]=\operatorname{det}\left[\widehat{I}^{(q)}+2 \rho x \widehat{\Delta}^{(q)} \widehat{B}^{(q)}\right]
$$

9. Consider now target group of $z=y+1$ replicas. In this case, $\widehat{B}_{\gamma}^{(q)}=\widehat{B}_{p}^{(q)}$ for $\gamma=1$ and $\widehat{B}_{\gamma}^{(q)}=\widehat{B}^{(q)}$ otherwise. We write therefore

$$
\begin{aligned}
\widehat{I}^{(z q)} & +\widehat{\lambda}^{(z)} \otimes \widehat{\Delta}^{(q)}\left(\widehat{\mathcal{R}}^{(z q)}\right) \widehat{B}^{(z q)}\left(\widehat{\mathcal{R}}^{(z q)}\right)^{-1}= \\
& =\widehat{I}^{(q)} \delta_{\alpha \beta}+\delta_{\alpha 1} \widehat{\Delta}^{(q)}\left(\widehat{B}_{p}^{(q)}-\widehat{B}^{(q)}\right)+(y+1) \delta_{\alpha 1} \delta_{1 \beta} \widehat{\Delta}^{(q)} \widehat{B}^{(q)}
\end{aligned}
$$

This is the block matrix of the peculiar form such that only upper block is nonzero in the first column; for that reason, its determinant is equal to the product of determinants of diagonal blocks (see Lemma 1). Thus,

$$
\operatorname{det}\left[\widehat{I}^{((y+1) q)}+2 \rho \widehat{\tilde{q}}^{(y+1)} \otimes \widehat{\Delta}^{(q)} \widehat{B}^{((y+1) q)}\right]=\operatorname{det}\left[\widehat{I}^{(q)}+2 \rho \widehat{\Delta}^{(q)}\left(y \widehat{B}^{(q)}+\widehat{B}_{p}^{(q)}\right)\right] .
$$

10. As to the second term in $\epsilon_{z}$ (A2), it is easily computed using Lemma 2. Indeed, $\widehat{B}^{((y+1) q)}$ is block diagonal matrix with one block $\widehat{B}_{p}^{(q)}$ and $y$ others $\widehat{B}^{(q)}$. On the other hand, $\widehat{\widetilde{q}}^{(y+1)} \otimes \widehat{\Delta}^{(q)}$ is the block matrix with every block being the same $\widehat{\Delta}^{(q)}$. Therefore, the matrix in question, $\left[\widehat{I}^{(z q)}+2 \rho \widehat{\tilde{q}}^{(z)} \otimes \widehat{\Delta}^{(q)} \widehat{B}^{(z q)}\right]$, is exactly of the form $\widehat{V}_{\widehat{g}, \widehat{h}}^{(z)}$ form, where $\widehat{g}=\widehat{\Delta}^{(q)} \widehat{B}_{p}^{(q)}$ and $\widehat{h}=\widehat{\Delta}^{(q)} \widehat{B}^{(q)}$. Using block matrix multiplication rule, it is easy to compute $\widehat{B}^{((y+1) q)} \widehat{V}_{\widehat{e}, \widehat{f}}^{(y+1)}$ (see Lemma 2) and then to use the result of Lemma 3. This finally gives

$$
\begin{aligned}
& \frac{1}{\rho}\langle\vec{\rho}| \widehat{B}^{(z q)}\left[\widehat{I}^{(z q)}+2 \rho \widehat{\tilde{q}}^{(z)} \otimes \widehat{\Delta}^{(q)} \widehat{B}^{(z q)}\right]^{-1}|\vec{\rho}\rangle= \\
& \quad=\rho\left\langle\vec{p}\left|\left(\widehat{B}_{p}^{(q)}+y \widehat{B}^{(q)}\right)\left[\widehat{I}^{(q)}+2 \rho y \widehat{\Delta}^{(q)} \widehat{B}^{(q)}+2 \rho \widehat{\Delta}^{(q)} \widehat{B}_{p}^{(q)}\right]^{-1}\right| \vec{p}\right\rangle
\end{aligned}
$$


11. Similar expression for a non-target group of $x$ replicas can be derived from here by formally putting $\widehat{B}_{p}^{(q)} \rightarrow \widehat{B}^{(q)}$ and $y+1 \rightarrow x$, this gives

$$
\frac{1}{\rho}\left\langle\vec{\rho}\left|\widehat{B}^{(z q)}\left[\widehat{I}^{(z q)}+2 \rho \widehat{\tilde{q}}^{(z)} \otimes \widehat{\Delta}^{(q)} \widehat{B}^{(z q)}\right]^{-1}\right| \vec{\rho}\right\rangle=\rho\left\langle\vec{p}\left|x \widehat{B}^{(q)}\left[\widehat{I}^{(q)}+2 \rho x \widehat{\Delta}^{(q)} \widehat{B}^{(q)}\right]^{-1}\right| \vec{p}\right\rangle
$$

\section{Lemma 1.}

Consider an auxiliary problem of the matrix

This is block matrix, where $\widehat{g}$ is $q \times q$ matrix and $\widehat{I}$ is identity matrix of the same size $q \times q$. The question is to find the determinant of this matrix.

It can be shown by expansion over the elements of the first column, then over the elements of the first column of the remaining minor, and by repeating this operation $q$ times, that

$$
\operatorname{det}\left[\widehat{U}_{\widehat{g}}^{(z)}\right]=\operatorname{det} \widehat{g}
$$

independently of the blocks placed in the upper-right triangle (shown conventionally with question marks).

\section{Lemma 2.}

Consider another auxiliary problem of the following block matrix:

Here $\widehat{g}$ and $\widehat{h}$ are matrices $q \times q$, they generally do not commute to each other. $\widehat{I}$ is identity matrix of the same size $q \times q$. Total size of the block matrix $\widehat{V}_{\widehat{g}, \widehat{h}}^{(z)}$ is, therefore, $z q \times z q$. The question is to find inverse of the matrix $\widehat{V}_{\widehat{g}, \widehat{h}}^{(z)}$.

It turns out that this inverse is in fact the matrix of the same structure, namely

$$
\begin{aligned}
& \left(\widehat{V}_{\widehat{g}, \widehat{h}}^{(z)}\right)^{-1}=\widehat{V}_{\widehat{e}, \widehat{f}}^{(z)} \text {, where } \\
& \qquad \widehat{e}=-(\widehat{I}+(z-1) \widehat{h}+\widehat{g})^{-1} \widehat{g} \text { and } \widehat{f}=-(\widehat{I}+(z-1) \widehat{h}+\widehat{g})^{-1} \widehat{h}
\end{aligned}
$$

The result can be easily proved using block matrix multiplication rule.

\section{Lemma 3.}

Consider an auxiliary problem of the scalar product 


$$
\left\langle\vec{\rho}^{(q z)}\left|\widehat{W}^{(q z)}\right| \vec{\rho}^{(q z)}\right\rangle
$$

where $\vec{\rho}^{(q z)}=\vec{p}^{(q)} \otimes \vec{i}^{(z)}=p_{i}$ (does not depend on replica indices $\alpha$ ), and $\widehat{W}^{(q z)}$ is block matrix comprised of blocks $\widehat{W}_{\alpha \beta}^{(q)}$. Obviously, this scalar product is reduced to the scalar products of smaller dimensionality $q$, that is, purely in species space, summed over all the blocks of the matrix:

$$
\left\langle\vec{\rho}^{(q z)}\left|\widehat{W}^{(q z)}\right| \vec{\rho}^{(q z)}\right\rangle=\left\langle\vec{p}^{(q)}\left|\left(\sum_{\alpha \beta} \widehat{W}_{\alpha \beta}^{(q)}\right)\right| \vec{p}^{(q)}\right\rangle .
$$




\section{APPENDIX B: RELATIONSHIP BETWEEN THE AVERAGE NUMBER OF SPECIES-SPECIES CONTACTS AND THE INTERACTION MATRIX}

Note that this relation can be easily derived directly from our formalism as well: The Hamiltonian (1) can also be expressed directly in terms of the number of contacts $n_{i j}$ between monomers of species $i$ and $j: \mathcal{H}=\sum_{i j}^{q} B_{i j} n_{i j}$, where we have previously substituted $n_{i j}=$ $\sum_{I \neq J}^{N} \delta_{s_{I}, i} \delta_{s_{J}, j} \delta\left(\mathbf{r}_{I}-\mathbf{r}_{J}\right)$.

Therefore, the average number of contacts can be directly calculated in terms of the derivative of the free energy with respect to $B_{i j}$. However, at this point, we must indicate one point in which we have been a bit cavalier in our previous derivation. Specifically, in order to perform the Hubbard-Stratonovich transformation, we have summed over all pairs of monomers $\sum_{I, J}$ instead of only the different pairs $\sum_{I \neq J}$. This overcounting of self-site interaction leads to a spurious term in the free energy $\widehat{\Delta} \widehat{B}$. Excluding this term from the free energy, which is equivalent to performing the sum $\sum_{I \neq J}$, carrying terms in the free energy to $\mathcal{O}\left(B^{2}\right)$, and taking the derivative with respect to $B_{i j}$ yields

$$
\left\langle n_{i j}\right\rangle=p_{i} p_{j}\left(1-\frac{B_{i j}}{T_{m}}\right) \approx p_{i} p_{j} \exp \left(-\frac{B_{i j}}{T_{m}}\right)
$$

of Ref. [27], i.e. either $T_{m}=T_{p}$ for chains in the target phase, $T_{m}=T_{f}$ for chains in the frozen phase, or $T_{m}=T$ for chains in the random phase. 


\section{REFERENCES}

[1] J. D. Bryngelson, J. Chem. Phys. 100, 6038 (1994).

[2] E.I. Shakhnovich and A.M. Gutin, Proc. Nat. Acad. Sci., USA 90, 7195 (1993).

[3] V. S. Pande, A. Yu. Grosberg, and T. Tanaka, J. Chem. Phys. 101, 8246 (1994).

[4] K.Yue and K. A. Dill, Proc. Nat. Acad. Sci., USA 89, 4163 (1992).

[5] J. D. Bryngelson and P. G. Wolynes, Proc. Nat. Acad. Sci., USA 84, 7524 (1987).

[6] P.G. Wolynes, in Spin Glass Ideas in Biology, edited by D. Stein (World Scientific, Singapore, 1991).

[7] T. Garel and H. Orland, Europhys. Lett. 6, 307 (1988).

[8] E. Shakhnovich, and A. Gutin, Biophys. Chem. 34, 187 (1989).

[9] C. Sfatos, A. Gutin, and E. Shakhnovich, Phys. Rev. E. 48, 465 (1993).

[10] V. S. Pande, A. Yu. Grosberg, and T. Tanaka, Phys. Rev. E. 51, 3381 (1995).

[11] H. Abe, N.Go, Biopolymers 20, 1013 (1981).

[12] O. B. Ptitsyn and M. V. Volkenstein, Journal of Biomolecular Structure and Dynamics, 4137 (1986).

[13] V. S. Pande, A. Yu. Grosberg, and T. Tanaka, Proc. Nat. Acad. Sci. USA 91, 12976 (1994)

[14] V. S. Pande, A. Yu. Grosberg, and T. Tanaka, J. Phys. II (Paris) 4, 1771 (1994).

[15] S. Ramanathan and E. I. Shakhnovich, Phys. Rev. E 72, 3907 (1994).

[16] V. S. Pande, A. Yu. Grosberg, and T. Tanaka, Macromolecules 28, 2218 (1995).

[17] E. I. Shakhnovich and A. M. Gutin, J. Theor. Biol 149, 537 (1991). 
[18] This Russian sentence says: "For example, to the best of our hope, our reader considers this text to be written in English and thus unlikely understands this sentence".

[19] K.Yue, K. M. Fiebig, P. D. Thomas, H. S. Chan, K. A. Dill, and E. I. Shakhnovich, Proc. Nat. Acad. Sci, USA 92, 325 (1995).

[20] V. S. Pande, A. Yu. Grosberg, and T. Tanaka, J. Phys. A, in press (1995).

[21] D. J. Amit, H. Gutfreund, and H. Sompolinsky, Annals of Physics 173, 30 (1987).

[22] M. Doi, M. and S. F. Edwards, Theory of Polymer Dynamics Clarendon Press, Oxford (1986)

[23] I. M. Lifshits, A. Yu. Grosberg, A. R. Kokhkolov, Rev. Mod. Phys. 50, 683 (1978).

[24] B. Derrida, Phys. Rev. Lett 45, 79 (1980).

[25] S. Miyazawa and R. Jernigan, Macromolecules 18, 534 (1985).

[26] R. A. Goldstein, Z. A. Luthey-Schulten, , P. G. Wolynes, Proc. Nat. Acad. Sci., USA 89, 4918 (1992).

[27] Finkelstein, A. V., Gutin, A. M., Badretdinov, A. Y., Sub-cellular biochemistry 24, 1 (1995). 
FIGURES

FIG. 1. Phase diagram for different values of the matrix similarity factor $g$. We find three phases for designed globular heteropolymers: Random, in which many $\left(\mathcal{O}\left(e^{N}\right)\right)$ conformations dominate equilibrium much as the equilibrium conformation of a globular homopolymer; Frozen, in which only a few $(\mathcal{O}(1))$ conformations dominate equilibrium in a glass-like phase; and Target in which only the target conformation (Native state) is found. For decreasing values of $g$, the boundary of the target phase moves to the left. For example, consider the case in which one performs a computer simulation of protein folding: nature has "prepared" proteins with the matrix of interactions $\widehat{B}^{p}$ at some preparation temperature $T_{p} / T_{p f}<1$ and one wishes to renature these proteins with some simulated potentials $\widehat{B}$ at some simulated acting temperature $1<T / T_{f}<T_{\operatorname{tar}} / T_{f}$; this desired pair of prepatation and acting temperatures is signified by a circle on the figure. If one could exactly reproduce the potentials used in Nature, i.e. $\widehat{B}=\widehat{B}^{p}$, then $g=1$ and the phase behavior is unchanged from Naturally renatured proteins: the circle is within the target phase. If the potentials used for renaturation are not precisely those used for preparation, i.e. $g<1$, then these errors in the potentials effect the nature of the phases by moving the boundary of the target phase to the left, thereby shrinking the target phase. If the errors are small compared with the degree of optimization, renaturation to the target phase is still possible, i.e. as shown in the figure, the circle is still in the target phase for $g>0.95$. Physically, the optimization of the native state ("preparation") allows some errors and this simply leads to a less optimized native state. Once these errors are large enough to overcome the optimization, the system will no longer renature to the target phase: in the figure we see that for $g<0.95$, the circle is no longer in the target phase. 


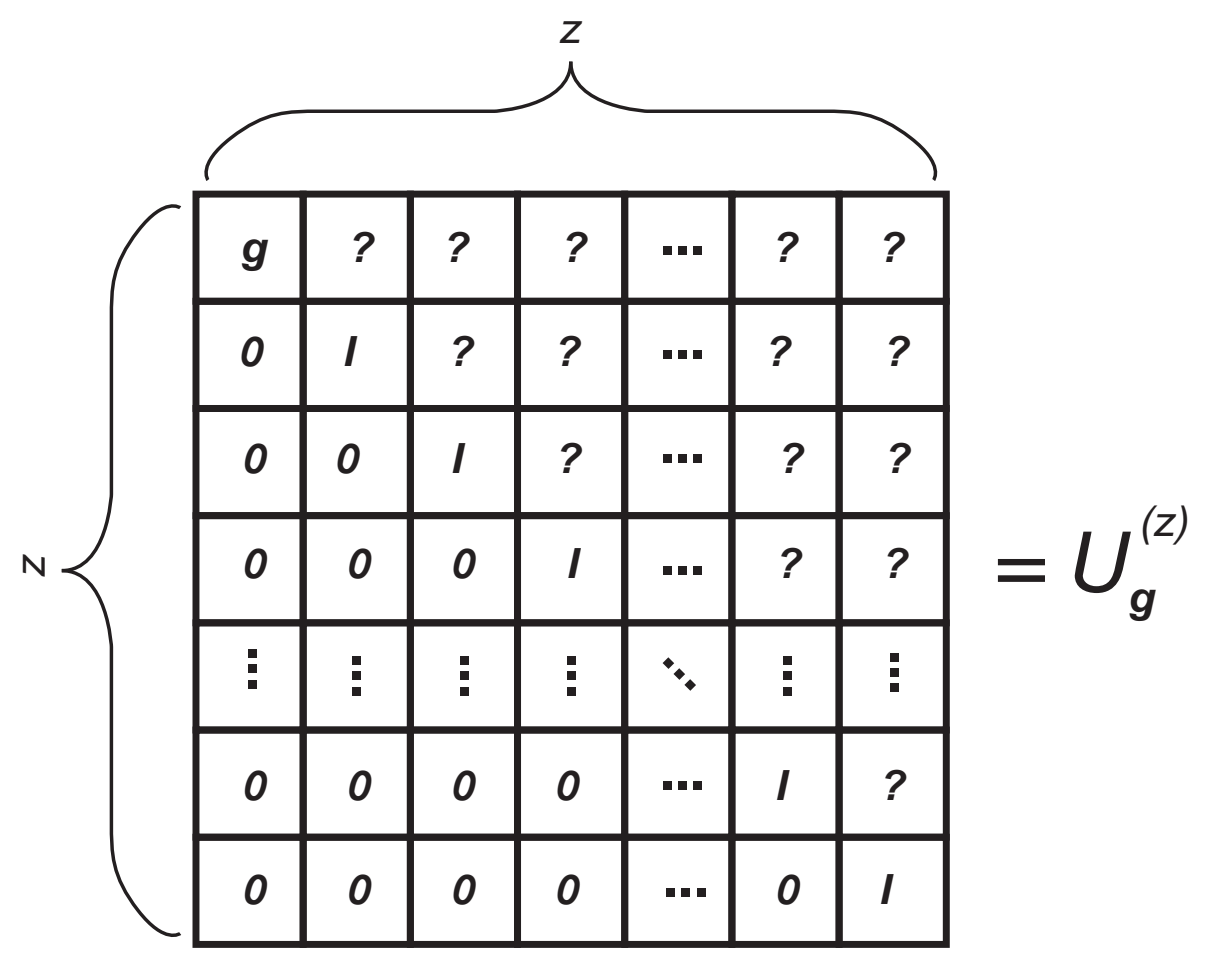




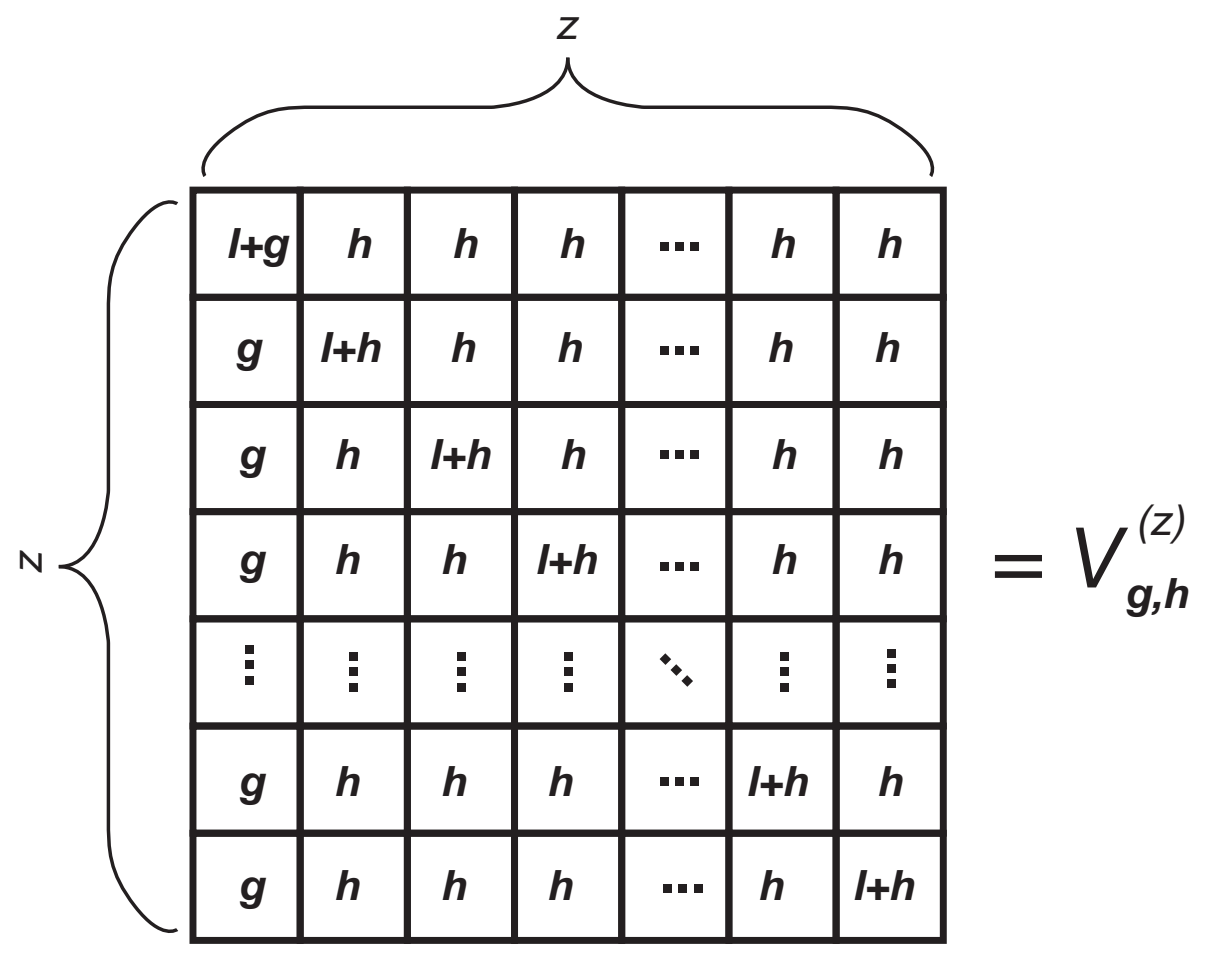




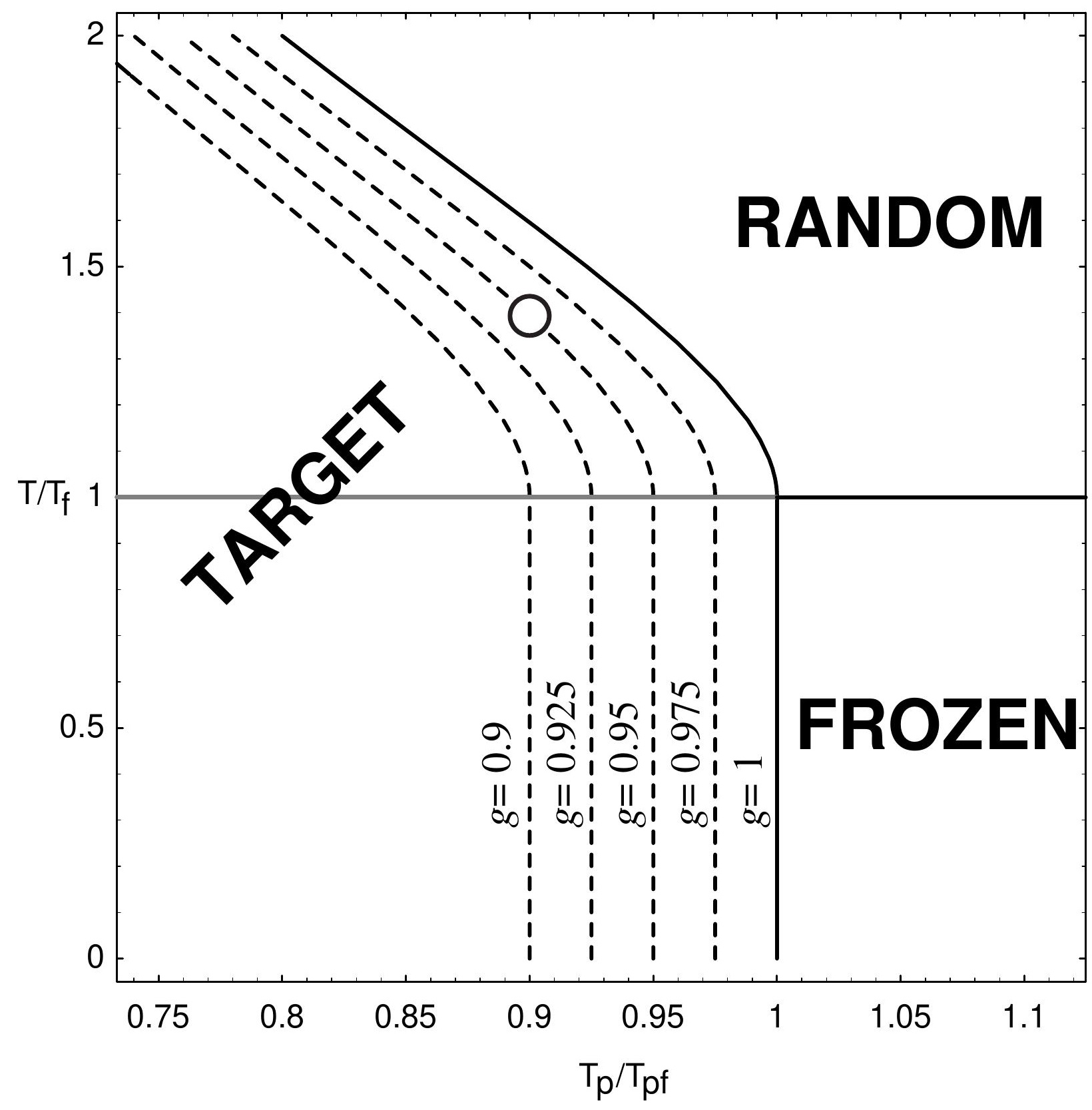

